\title{
Cinética de secagem, contração volumétrica e análise da difusão líquida do figo (Ficus carica L.)
}

\author{
Luiz C. Corrêa Filho' ${ }^{1}$, Ednilton T. de Andrade ${ }^{2}$, Ana P. Martinazzo ${ }^{3}$, \\ Elisabeth M. D’Andrea ${ }^{1}$, Felipe A. de Sousa ${ }^{1} \&$ Vitor G. Figueira ${ }^{1}$ \\ ${ }^{1}$ Escola de Engenharia de Niterói/Universidade Federal Fluminense. Niterói, RJ. E-mail: lucaalbernaz@gmail.com (Autor correspondente); \\ elisabethmdandrea@gmail.com; felipesousa@id.uff.br; figueiravitor@yahoo.com.br \\ ${ }^{2}$ Departamento de Engenharia Agrícola/Universidade Federal de Lavras. Lavras, MG. E-mail: ednilton@deg.ufla.br \\ ${ }^{3}$ Departamento Engenharia de Agronegócios/Escola de Engenharia de Volta Redonda/Universidade Federal Fluminense. Volta Redonda, RJ. E-mail: \\ anamartinazzo@metal.eeimvr.uff.br
}

\section{Palavras-chave:}

modelagem matemática pós-colheita

fruta

\begin{abstract}
R E S U M O
A secagem é um processo utilizado para retirar certa quantidade de água do produto com a finalidade de aumentar a vida de prateleira, minimizar as perdas na pós-colheita, do excesso da produção e devido a contração volumétrica reduzir os custos de embalagem, transportes e armazenamento. Diante do exposto objetivou-se no trabalho fazer o estudo da cinética de secagem, análise da contração volumétrica e obter os valores de difusão líquida durante o processo de secagem de figo. Foram utilizados figos do comércio da cidade de Niterói, RJ, e submetidos à secagem em condições controladas de temperaturas do ar $\left(40,55\right.$ e $\left.70{ }^{\circ} \mathrm{C}\right)$. Aos dados experimentais foram ajustados modelos matemáticos para a representação do processo de contração volumétrica e de secagem de produtos agrícolas. $\mathrm{O}$ modelo polinomial teve o melhor ajuste aos dados observados para a faixa de temperatura estudada representando melhor a contração volumétrica do figo e para secagem o modelo de Midilli obteve o melhor ajuste. A difusão líquida aumenta com a elevação da temperatura apresentando valores entre $1,76 \times 10^{-11}$ e $8,78 \times 10^{-11} \mathrm{~m}^{2} \mathrm{~s}^{-1}$.
\end{abstract}

\section{Key words:} mathematical modeling post-harvest fruit

\section{Drying kinetics, shrinkage and analysis of liquid diffusion of figs}

\begin{abstract}
A B S T R A C T
The drying process is used to remove some water from the product, in order to increase the shelf life, to minimize post-harvest losses in excess of production and owing to the shrinkage, reducing the cost of packaging, transport and storage. Thus, the objective of this work was to study the drying of fig, analysis of shrinkage, as well as obtaining the values of liquid diffusion during the fig drying process. Figs were used from some Niterói Markets and subjected to drying under controlled air temperatures $\left(40,55\right.$ and $\left.70{ }^{\circ} \mathrm{C}\right)$. Mathematical models were adjusted to experimental data to represent the processes of shrinkage and drying of agricultural products. The polynomial model and Midilli had the best fit for the observed data for the temperature range studied, representing the best shrinkage and drying fig, respectively. The liquid diffusion increases with increasing temperature with values between $1.76 \times 10^{-11}$ and $8.78 \times 10-11 \mathrm{~m}^{2} \mathrm{~s}^{-1}$.
\end{abstract}




\section{INTRODUÇÃo}

A figueira (Ficus carica L.) é uma espécie originária da Ásia Menor e da Síria, na região mediterrânea, sendo considerada uma das mais antigas frutíferas cultivadas no mundo e que vem atravessando os séculos como alimento de diferentes povos. É classificada na ordem Urticales e pertencente à família das Moraceas e subfamilia Artocarpoideas que produz fruto com suco leitoso. Dentre as 2000 espécies presentes no gênero Fícus, a Ficus carica é a única que tem valor econômico. Apresenta excelente adaptação a diferentes climas e é cultivada tanto em regiões subtropicais quentes como em climas temperados (Simão, 1971; Sousa, 2008; Rodrigues et al., 2012).

O figo, fruto da figueira, é reconhecido como antioxidante, laxante, diurético, digestivo, com ação benéfica para o fígado e depurativo do sangue. O figo seco, cozido com água ou leite, é béquico e expectorante (Pacco et al., 2007). Segundo Doymaz (2005), o figo tem grande importância na nutrição por ser fonte inesgotável de carboidratos e conter aminoácidos essenciais, vitaminas A, B1, B2 e C.

Leonel \& Tecchio (2010) relatam que a cultura da figueira é interessante para o Brasil haja vista que, atualmente, é o segundo maior exportador de frutos in natura do mundo, cuja produção coincide com a entressafra do mercado mundial, no hemisfério Norte. A produção brasileira está concentrada na região de Valinhos-Campinas, estado de São Paulo, com cultivo caracterizado como atividade tipicamente familiar, responsável por $98 \%$ da produção do estado (Francisco et al., 2011).

O figo é uma fruta altamente perecível, o que ocasiona grandes perdas durante a colheita; portanto, a minimização dessas perdas é de grande interesse sendo o processo de secagem uma alternativa bastante utilizada na indústria de alimentos para preservação de frutas por ter, como objetivo, reduzir a atividade de água, inibir o crescimento microbiano, a atividade enzimática tal como as mudanças físicas e químicas do alimento durante o armazenamento possibilitando, assim, o aumento de sua vida de prateleira e reduzindo custos de transporte, armazenamento e embalagem (Sousa, 2008).

Devido ao seu alto teor em açúcar, o figo seco foi utilizado durante décadas como suplemento alimentar nos tempos difíceis das duas Guerras Mundiais e na fabricação de álcool. O resíduo do figo destilado era utilizado na alimentação animal (Pacco et al., 2007).

A secagem solar é o método convencional usado para obtenção de figos secos, o qual requer baixo custo e equipamentos simples além de gerar produto com boas propriedades sensoriais; contudo, a secagem mecânica tem ganhado importância por apresentar vantagens como: melhores condições sanitárias, reduzindo contaminação pela exposição da fruta e parâmetros de secagem controlados proporcionando produtos mais uniformes e com menor degradação de qualidade (Sousa, 2008).

Um dos principais fatores relacionados à perda de qualidade de alimentos desidratados está pautado nas alterações estruturais favorecidas pelo encolhimento, também denominado contração volumétrica, durante a secagem. Uma das mudanças físicas mais importantes para melhor entendimento do processo de secagem em alimentos é o encolhimento e, consequentemente, alterações na sua densidade aparente podendo ser muito intensa dependendo do método de secagem aplicado afetando, em particular, o coeficiente de difusão mássica do material, que é um dos parâmetros fundamentais que regem o processo de secagem, mas também tem influência sobre a taxa de secagem (Krokida \& Maroulis, 1997; Lima et al., 2002; Goneli et al., 2011).

Quando desidratados, materiais biológicos porosos se contraem diferentemente nas direções longitudinal, tangencial e radial. Mudanças das características dimensionais dos produtos, devido à sua desidratação, são relatadas como as primeiras causas das alterações das principais propriedades físicas de produtos agrícolas. Este encolhimento é um fenômeno característico da secagem de produtos agrícolas sobremaneira os que possuem alto teor de água; assim, é essencial considerá-lo para a previsão de perfis de teor de água do produto, visto que a cinética de desidratação é, também, uma função da temperatura do ar de secagem, podendo resultar em produtos com volumes diferentes; desta forma, a obtenção da cinética de contração para diferentes temperaturas do ar de secagem e teores de água se torna importante no conhecimento das características intrínsecas do produto.

A partir do exposto o objetivo no presente trabalho foi analisar a cinética de secagem em conjunto com a contração volumétrica do figo, estimar e analisar as curvas de secagem e contração, ajustar modelos matemáticos aos valores experimentais que representem cada processo e determinar o coeficiente de difusão líquida para a faixa de temperatura estudada.

\section{Material e Métodos}

O presente trabalho foi desenvolvido no Laboratório de Termociências (LATERMO) localizado na Escola de Engenharia no campus da Praia Vermelha da Universidade Federal Fluminense, em Niterói, estado do Rio de Janeiro, entre Junho e Julho de 2013.

Utilizaram-se, como matéria-prima, figos frescos adquiridos no comércio do município de Niterói, RJ, os quais foram cortados longitudinalmente para determinação das curvas de secagem e contração volumétrica. O teor de água inicial foi determinado gravimetricamente, por perda de massa em estufa a $105^{\circ} \mathrm{C}$ variando de $\pm 1{ }^{\circ} \mathrm{C}$ durante $24 \mathrm{~h}$ (IAL, 1985).

Foram avaliadas diferentes temperaturas do ar de secagem $\left(40,55\right.$ e $\left.70^{\circ} \mathrm{C}\right)$ combinadas com a velocidade do ar de secagem de $1,2 \mathrm{~m} \mathrm{~s}^{-1}$. O secador utilizado é um protótipo com controle de temperatura e vazão constante, composto por bandejas removíveis com fundo telado, dispostas horizontalmente para permitir a passagem do ar por entre o produto; a vazão do ar de secagem foi medida por meio de um anemômetro digital enquanto a temperatura do secador foi obtida e controlada por meio de um termômetro ordinário, com precisão de $0,1^{\circ} \mathrm{C}$.

Em cada tratamento de secagem a umidade relativa e a temperatura do ar foram registradas por um termohigrógrafo, e a umidade relativa do ar secante foi calculada pelas equações psicrométricas, por meio de um programa computacional denominado Grapsi.

$\mathrm{Na}$ obtenção das curvas de secagem avaliou-se a perda de água ao longo da secagem calculada por diferença de massa, 
pesando-se as bandejas em intervalos regulares de tempo, até o produto atingir o teor de água de equilíbrio.

Para o cálculo da razão de umidade (RU), foi calculado o teor de água de equilíbrio de figos em diferentes umidades relativas e níveis de temperatura usando-se o modelo de GAB (Guggenheim-Anderson-De Boer) descrito pela Eq. 1 (Anderson, 1946; Boer, 1953; Guggenheim, 1966; Berg, 1984):

$$
\mathrm{M}_{\mathrm{eq}}=\frac{\mathrm{M}_{\mathrm{m}} \cdot \mathrm{c} \cdot \mathrm{k} \cdot \alpha_{\mathrm{w}}}{\left(1-\mathrm{k} \alpha_{\mathrm{w}}\right) \cdot\left(1-\mathrm{k} \alpha_{\mathrm{w}}+\mathrm{ck} \alpha_{\mathrm{w}}\right)}
$$

onde os coeficientes são dados pelas Eqs. 2 e 3:

$$
\begin{aligned}
& c \quad \exp \left(\frac{}{R T}\right) \\
& k=k_{0} \exp \left(\frac{\Delta H_{k}}{R_{g} T}\right)
\end{aligned}
$$

em que:

$\mathrm{M}_{\mathrm{eq}}$ - teor de água de equilíbrio, decimal b.s;

$\mathrm{M}_{\mathrm{m}}$ - teor de água na monocamada molecular;

$\mathrm{c}, \mathrm{k}, \mathrm{c}_{0}, \mathrm{k}_{0}$ - constantes da equação de GAB, adimensional;

$\alpha_{w} \quad$ - atividade de água, decimal;

$\mathrm{R}_{\mathrm{g}}$ - constante universal do gás, $\mathrm{J} \mathrm{mol}^{-1} \mathrm{~K}^{-1}$; e

$\mathrm{T}^{\mathrm{g}}$ - temperatura do ar, ${ }^{\circ} \mathrm{C}$.

No cálculo do teor de água de equilíbrio foram assumidos os seguintes valores para as constantes, propostos por MarinosKouris \& Maroulis (1995): $\mathrm{M}_{\mathrm{m}}=11,7 \%$; $\mathrm{c}_{0}=1,77, \mathrm{H}_{\mathrm{c}}=-1,55 \mathrm{~J}$ $\mathrm{mol}^{-1} \mathrm{~K}^{-1}, \mathrm{k}_{0}=0,05, \mathrm{H}_{\mathrm{k}}=25,2 \mathrm{~J} \mathrm{~mol}^{-1} \mathrm{~K}^{-1}$ e a constante universal do gás $\mathrm{Rg}=8,3143 \mathrm{~J} \mathrm{~mol}^{-1} \mathrm{~K}^{-1}$

Para cálculo da atividade de água $\left(\mathrm{a}_{\mathrm{w}}\right)$ utilizou-se a umidade relativa do ar secante obtida pelo software Grapsi versão 6 na Eq. 4:

$$
\mathrm{a}_{\mathrm{w}}=\frac{\mathrm{UR}}{100}
$$

Aos dados experimentais de secagem das amostras de figos foram ajustados os modelos matemáticos representados pelas Eqs. de 5 a 15, respectivamente, conforme Tabela 1.

Tabela 1. Modelos matemáticos para ajuste das curvas de secagem do figo

\section{Modelo} Equações

Aproximação da difusão $R U=a \cdot \exp (-k \cdot t)+(1-a) \cdot \exp (-k \cdot b \cdot t)$

Dois Termos $\quad \mathrm{RU}=\mathrm{a} \cdot \exp \left(-\mathrm{k}_{0} \cdot \mathrm{t}\right)+\mathrm{b} \cdot \exp \left(-\mathrm{k}_{1} \cdot \mathrm{t}\right)$

Exponencial de 2 termos $\mathrm{RU}=\mathrm{a} \cdot \exp (-\mathrm{k} . \mathrm{t})+(1-\mathrm{a}) \exp (-\mathrm{k} \cdot \mathrm{a} \cdot \mathrm{t})$

Logaritmo $\quad \mathrm{RU}=\mathrm{a} \cdot \exp (-\mathrm{k} \cdot \mathrm{t})+\mathrm{b}$

Midili $\quad \mathrm{RU}=\mathrm{a} \cdot \exp \left(-\mathrm{k} \cdot \mathrm{t}^{\mathrm{n}}\right)+\mathrm{b} \cdot \mathrm{t}$

Newton $\quad \mathrm{RU}=\exp (-\mathrm{k} . \mathrm{t})$

Page $\quad \mathrm{RU}=\exp \left(-\mathrm{k} . \mathrm{t}^{\mathrm{n}}\right)$

Page modificado $\quad \mathrm{RU}=\exp \left[-(\mathrm{k} . \mathrm{t})^{\mathrm{n}}\right]$

Thompson $\quad \mathrm{RU}=\exp \left\{\left[-\mathrm{a}-\left(\mathrm{a}^{2}+4 \cdot \mathrm{b} \cdot \mathrm{t}\right)^{0,5}\right] / 2 \cdot \mathrm{b}\right\}$

Verna $\quad \mathrm{RU}=\mathrm{a} \cdot \exp (-\mathrm{k} \cdot \mathrm{t})+(1-\mathrm{a}) \exp (-\mathrm{k} 1 . \mathrm{t})$

Wang e Singh $\quad \mathrm{RU}=1+\mathrm{at}+\mathrm{bt}^{2}$

$\mathrm{RU}$ - Razão de umidade (adimensional); $\mathrm{k}, \mathrm{k}_{0}, \mathrm{k}_{1}$ - Constantes de secagem (s $\left.\mathrm{s}^{-1}\right)$; $\mathrm{t}$ - Tempo de secagem, em h; a, b, $\mathrm{n}$ - Constantes que dependem da natureza do produto, adimensional (Brooker et al., 1992; Corrêa et al., 2001; Alves et al., 2013)
Para o ajuste dos modelos matemáticos aos dados experimentais das curvas de secagem foi utilizada análise de regressão não linear, pelo método Quasi-Newton, por meio do programa Statistica 7.0. Os dados experimentais foram comparados com os valores calculados pelos modelos, por meio dos erros médio relativo (P), estimado (SE) e do Qui Quadrado $\left(\chi^{2}\right)$, conforme descrito nas Eqs. 16, 17 e 18, respectivamente. A capacidade do modelo em descrever com fidelidade o processo físico é inversamente proporcional ao valor de SE (Chen \& Morey, 1989; Chen \& Jayas, 1998).

$$
\begin{aligned}
& \mathrm{P}=\frac{100}{\mathrm{n}} \cdot \sum \frac{|\mathrm{Y}-\hat{\mathrm{Y}}|}{\mathrm{Y}} \\
& \mathrm{SE}=\sqrt{\frac{\sum(\mathrm{Y}-\hat{\mathrm{Y}})^{2}}{\mathrm{GLR}}} \\
& \chi^{2}=\frac{\sum(\mathrm{Y}-\hat{\mathrm{Y}})^{2}}{\mathrm{GLR}}
\end{aligned}
$$

em que:

Y - valor observado experimentalmente;

Y - valor calculado pelo modelo; e

GLR - graus de liberdade do modelo.

De acordo com Brooker et al. (1992), o modelo de difusão líquida pode ser descrito segundo a lei de Fick (Eq. 19) que tem, como objetivo, estudar o comportamento da difusão da água em função do gradiente de concentração; para materiais homogêneos considera-se a Eq. 20.

$$
\begin{gathered}
\frac{\partial \mathrm{U}^{*}}{\partial \mathrm{t}}=\frac{\partial}{\partial \mathrm{x}}\left(\mathrm{D} \frac{\partial \mathrm{U}^{*}}{\partial \mathrm{x}}\right) \\
\frac{\partial \mathrm{U}^{*}}{\partial \mathrm{t}}=\mathrm{D}\left[\frac{\partial^{2} \mathrm{U}^{*}}{\partial \mathrm{x}^{2}}+\frac{\mathrm{c}}{\mathrm{x}} \frac{\partial \mathrm{U}^{*}}{\partial \mathrm{x}}\right]
\end{gathered}
$$

em que:

$\mathrm{U}^{*} \quad$ - teor de água do produto, decimal b.s;

D - coeficiente de difusão líquida, $\mathrm{m}^{2} \mathrm{~s}^{-1}$;

t - tempo, s;

x - distância em relação a um ponto de referência no corpo, $\mathrm{m} ; \mathrm{e}$

c - 0 para parede infinita, 1 para corpos cilíndricos e 2 para corpos esféricos.

No cálculo da razão de umidade (RU), durante a secagem nas diferentes condições de ar foi utilizada a Eq. 21 para a forma geométrica do tipo esfera proposto por Brooker et al. (1992).

$$
R U=\frac{U-U_{e}}{U_{i}-U_{e}}=\frac{6}{\pi^{2}} \sum_{n=1}^{\infty} \frac{1}{n^{2}} \exp \left[-\frac{n^{2} \cdot \pi^{2} \cdot D \cdot t}{R^{2}} .\right]
$$

em que: 
RU - razão de umidade do produto, adimensional;

$\mathrm{U}$ - teor de água do produto, decimal b.s;

Ue - teor de água de equilíbrio de produto, decimal b.s;

$\mathrm{Ui}$ - teor de água inicial do produto, decimal b.s;

n - número de termos;

D - coeficiente de difusão líquida, $\mathrm{m}^{2} \mathrm{~s}^{-1}$;

t - tempo, s; e

$\mathrm{R}$ - raio equivalente, $\mathrm{m}$.

A taxa de redução de água durante a secagem de figo foi determinada por (Corrêa et al., 2001) que a definem como a quantidade de água que determinado produto perde por unidade de matéria seca do produto por unidade de tempo, descrito na Eq. 22:

$$
\operatorname{TRA}=\frac{\left(\mathrm{Ma}_{0}-\mathrm{Ma}_{\mathrm{i}}\right)}{\mathrm{M}_{\mathrm{S}}\left(\mathrm{t}_{\mathrm{i}}-\mathrm{t}_{0}\right)}
$$

em que:

TRA - taxa de redução de água, $\mathrm{kg} \mathrm{kg}^{-1} \mathrm{~h}^{-1}$;

$\mathrm{Ma}_{\mathrm{o}}$ - massa de água total atual, kg;

$\mathrm{Ma}_{\mathrm{i}}$ - massa de água total anterior, kg;

Ms - matéria seca, kg;

$\mathrm{t}_{\mathrm{o}} \quad$ - tempo total de secagem anterior, h; e

$t_{i} \quad$ - tempo total de secagem atual, $h$.

Para a determinação do volume o figo foi considerado um cone elíptico sendo medidas suas dimensões por meio de paquímetro digital com resolução de 0,01 m. Utilizou-se, para determinar o volume, a Eq. 23 enquanto o índice de contração volumétrica do figo $(\Psi)$, durante a secagem foi determinado pela relação entre o volume para cada teor de água e o volume inicial, de acordo com a Eq. 24 (Mohsenin, 1986):

$$
\begin{gathered}
V=\frac{\pi(a b c)}{6} \\
\Psi=\frac{V}{V_{0}}
\end{gathered}
$$

em que:

$\mathrm{V} \quad$ - volume em cada teor de água, $\mathrm{m}^{3}$;

a - comprimento em $\mathrm{m}^{3}$;

b - espessura em $\mathrm{m}^{3}$;

c - profundidade em $\mathrm{m}^{3}$;

$\Psi \quad$ - índice de contração volumétrica, decimal;

$\mathrm{V}$ - volume em cada teor de água, $\mathrm{m}^{3}$; e

$\mathrm{V}_{0} \quad$ - volume inicial, $\mathrm{m}^{3}$.

Nos modelos matemáticos, descritos na Tabela 2, as Eqs. 25 a 28 são adaptações em função da temperatura enquanto as Eqs. 29 até 33 foram, em função do teor de água, ajustadas aos dados experimentais de contração volumétrica $(\Psi)$.

Para o ajuste dos modelos matemáticos de contração volumétrica aos dados experimentais, foi realizada análise de regressão não linear. O grau de ajuste dos modelos aos dados experimentais foi avaliado conforme descrito anteriormente para as a cinética de secagem.
Tabela 2. Modelos matemáticos para ajuste das curvas de contração volumétrica do figo

\begin{tabular}{llr}
\multicolumn{1}{c}{ Modelo } & \multicolumn{1}{c}{ Equação } \\
Exponencial adaptado & $\Psi=\left(\mathrm{a}\left(\exp \left(\mathrm{bU}\left(\mathrm{T}^{\mathrm{c}}\right)\right)\right)\right)$ & $(25)$ \\
Linear adaptado & $\Psi=(\mathrm{a}+(\mathrm{bU})) \mathrm{T}^{\mathrm{c}}$ & $(26)$ \\
Rahman adaptado & $\Psi=\left(1+\left(\mathrm{a}\left(\mathrm{U}-\mathrm{U}_{0}\right)\right)\right) \mathrm{T}^{\mathrm{b}}$ & $(27)$ \\
Bala \& Woods adaptado & $\Psi=\left(1-\left(\mathrm{a}\left(1-\exp \left(-\mathrm{b}\left(\mathrm{U}_{0}-\mathrm{U}\right)\right)\right)\right)\right) \mathrm{T}^{\mathrm{c}}$ & $(28)$ \\
Correa & $\Psi=1 /(\mathrm{a}+\mathrm{b} \exp (\mathrm{U}))$ & $(29)$ \\
Polinomial & $\left.\Psi=(\mathrm{a}+\mathrm{bU}+\mathrm{CU})^{2}\right)$ & $(30)$ \\
Bala \& Woods modificado & $\Psi=1-\mathrm{a}\left\{1-\exp \left(\mathrm{b}\left(\mathrm{U}_{0}-\mathrm{U}\right)\right)\right\}$ & $(31)$ \\
Exponencial & $\Psi=\mathrm{A} \exp (\mathrm{b} \cdot \mathrm{U})$ & $(32)$ \\
Linear & $\Psi=\mathrm{a}+\mathrm{bU}$ & $(33)$ \\
\hline
\end{tabular}

$\Psi$ - Índice de contração volumétrica (decimal); $U$ - Teor de água (\% b.s.); $U_{0}$ - Teor de agua Inicial (\% b.s.); T - Temperatura do ar de secagem $\left({ }^{\circ} \mathrm{C}\right)$; a, b, c - Constantes que dependem do produto (Gonelli et al., 2011)

Para a determinação do modelo de difusão líquida os dados experimentais da contração volumétrica foram utilizados e os dados simulados foram obtidos por meio de regressão não linear, no programa Statistica 7.0 pelo método Quasi-Newton.

\section{Resultados e Discussão}

Dentre os onze modelos avaliados para predizer o fenômeno de secagem de figo e os nove modelos avaliados para a contração volumétrica, verificou-se que as equações de Midilli e a Polinomial foram as que melhor se ajustaram aos dados observados de secagem e contração volumétrica, respectivamente. Além das equações apresentarem os menores valores para o erro médio relativo $(\mathrm{P})$ e erro médio estimado (SE) e qui-quadrado $\left(\chi^{2}\right)$ oferecendo um ajuste melhor aos dados observados de razão de umidade, apresentaram também elevados valores do coeficiente de determinação $\left(R^{2}\right)$ estando para todas as temperaturas, acima de $98 \%$ mas apresentando, também, valores de erro médio relativo abaixo de $10 \%$, o que, segundo Madamba et al. (1996), significa um bom ajuste dos modelos para representação do fenômeno de secagem. Considera-se que valores de erro médio relativo abaixo de $10 \%$ indicam um ajuste razoável para as práticas propostas (Mohapatra \& Rao, 2005).

Em trabalhos com outros frutos o modelo de Midilli foi o que melhor representou o processo de secagem, como determinaram Paglarini et al. (2012) na secagem de abacaxi em secador de bandejas nas temperaturas de 40,50 e $60^{\circ} \mathrm{C}$ e o modelo Polinomial foi o que melhor se ajustou aos dados experimentais de contração volumétrica, como determinaram Goneli et al. (2011) na secagem em estufa com circulação forçada de ar dos frutos de mamona a $40{ }^{\circ} \mathrm{C}$ e Siqueira et al. (2012) na secagem convectiva de sementes de pinhão nas temperaturas de $36,45,60,75,90$ e $105^{\circ} \mathrm{C}$.

Nas Tabelas 3 e 4 são apresentados o qui-quadrado $\left(\chi^{2}\right)$, os erros médio estimados (SE) e relativo $(\mathrm{P})$ e coeficiente de determinação $\left(\mathrm{R}^{2}\right)$, para as temperaturas do ar de secagem de 40,55 e $70{ }^{\circ} \mathrm{C}$ do modelo de Midili em função dos dados observados na secagem e do modelo Polinomial em função dos dados observados de contração volumétrica, respectivamente.

Os teores de água inicial das amostras de figo submetidas ao processo de secagem nas temperaturas de 40,55 e $70{ }^{\circ} \mathrm{C}$ foram, respectivamente, 6,$92 ; 7,25$ e 8,43 b.s. Por meio dos resultados apresentados na Figura 1 observou-se o decréscimo do tempo 
Tabela 3. Constantes do modelo de Midili e os valores do qui quadrado $\left(\chi^{2}, x 100\right.$, adimensional), coeficiente de determinação $\left(\mathrm{R}^{2}, \%\right)$, erro médio estimado (SE, adimensional) e relativo ( $P$, adimensional) para as temperaturas estudadas.

\begin{tabular}{cccccccccc}
\hline Temp. & \multicolumn{4}{c}{ Parâmetros estatísticos } & & \multicolumn{4}{c}{ Constantes do modelo } \\
\cline { 2 - 4 } \cline { 7 - 9 }$\left({ }^{\circ} \mathbf{C}\right)$ & $\mathbf{X}^{2}$ & $\mathbf{P}$ & $\mathbf{S E}$ & $\mathbf{R}^{2}$ & $\mathbf{a}$ & $\mathbf{b}$ & $\mathbf{k}$ & $\mathbf{n}$ \\
40 & 0,003 & 5,785 & 0,005 & 99,97 & & 1,001 & $-0,002$ & 0,064 & 0,871 \\
55 & 0,008 & 5,69 & 0,009 & 99,92 & 0,978 & $-0,002$ & 0,073 & 1,055 \\
70 & 0,09 & 5,30 & 0,010 & 99,93 & 0,989 & $-0,012$ & 0,129 & 0,971 \\
\hline
\end{tabular}

Tabela 4. Constantes do modelo Polinomial e os valores do coeficiente de determinação $\left(R^{2}, \%\right)$, erro médio estimado ( $\mathrm{SE}$, adimensional) e relativo $(\mathrm{P}$, adimensional) e qui quadrado $\left(\chi^{2}\right.$, adimensional) do modelo para elaboração das curvas de contração volumétrica

\begin{tabular}{|c|c|c|c|c|c|c|c|}
\hline \multirow{2}{*}{$\begin{array}{l}\text { Temp. } \\
\left({ }^{\circ} \mathrm{C}\right)\end{array}$} & \multicolumn{4}{|c|}{ Parâmetros estatísticos } & \multicolumn{3}{|c|}{ Constante do modelo } \\
\hline & $\mathbf{P}$ & SE & $\mathrm{R}^{2}$ & $x^{2}$ & $a$ & b & C \\
\hline 40 & 8,6432 & 0,0435 & 98,01 & 0,00189 & 0,13705 & 0,00279 & 0,01482 \\
\hline 55 & 7,8027 & 0,0339 & 98,66 & 0,00115 & 0,11213 & 0,08859 & 0,00541 \\
\hline 70 & 3,3483 & 0,0269 & 99,01 & 0,000723 & 0,25041 & 0,05409 & 0,00458 \\
\hline
\end{tabular}

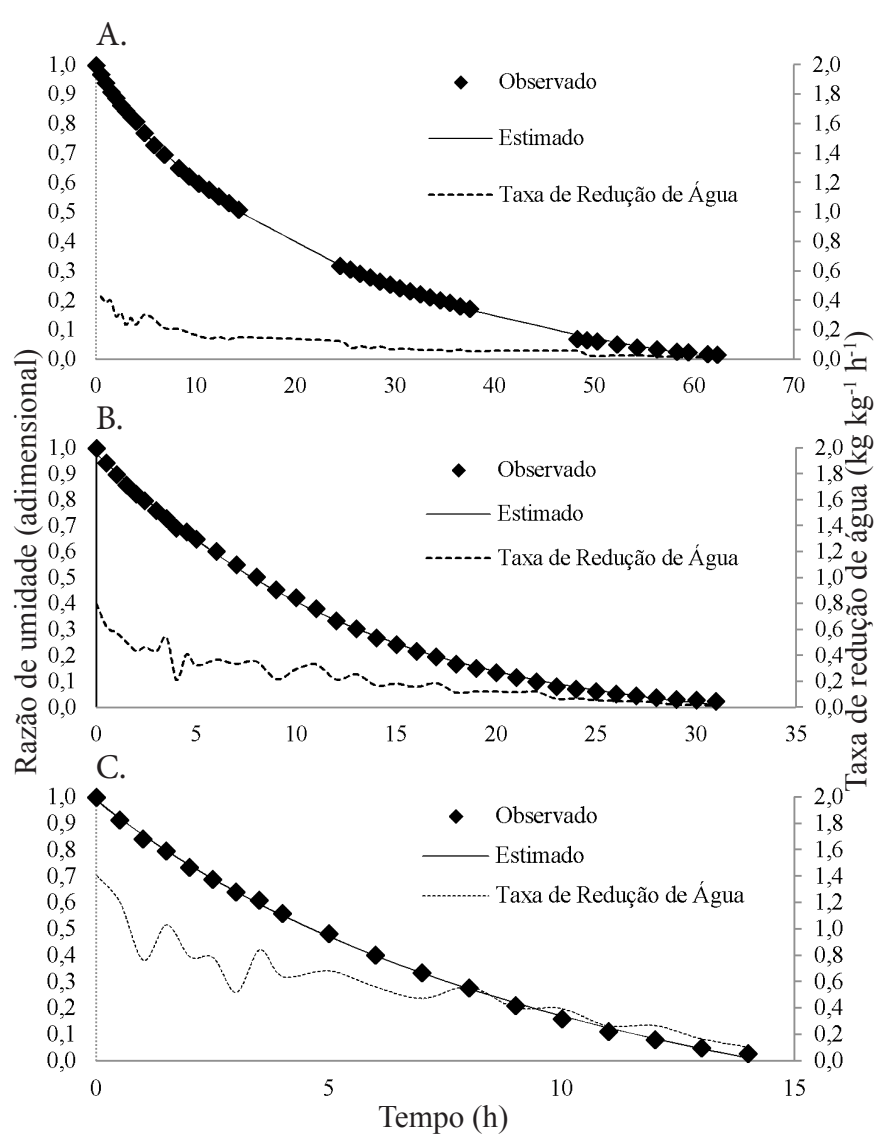

Figura 1. Curvas de razão de umidade observada e estimada pela equação de Midilli na secagem de figo para as temperaturas estudadas e taxa de redução de água, 40 (A); 50 (B) e $70{ }^{\circ} \mathrm{C}(\mathrm{C})$

de secagem com o aumento da temperatura do ar. O tempo médio necessário para completar o processo de secagem a 40 ${ }^{\circ} \mathrm{C}$ foi de $62,33 \mathrm{~h}$. Com um incremento de $15^{\circ} \mathrm{C}$ na temperatura inicial constatou-se uma redução de $50,3 \%$ do tempo de secagem $(31 \mathrm{~h})$. Na temperatura de $70^{\circ} \mathrm{C}$ o tempo de secagem foi reduzido em $77,5 \%$ em relação a $40^{\circ} \mathrm{C}$. Verificou-se, então, que a temperatura de secagem exerce influência significativa sobre a cinética de secagem do figo. Como observado por Borges et al. (2011) na secagem de bananas a 40 e $70{ }^{\circ} \mathrm{C}$ por convecção natural e Paglarini et al. (2012) na secagem de fatias de abacaxi variando a temperatura de 40 a $60{ }^{\circ} \mathrm{C}$ é possível observar também que a taxa de redução de água durante a secagem se apresentou homogênea sem variações excessivas para todas as temperaturas estudadas indicando que a secagem foi realizada de forma equilibrada.

Na Figura 2 são apresentados os valores experimentais de contração volumétrica para as temperaturas de 40,55 e $70{ }^{\circ} \mathrm{C}$ ajustados pelo modelo Polinomial, em função do tempo.

Analisando as curvas de contração volumétrica descrita na Figura 2, observa-se que os figos apresentaram redução de volume inicial de 84,04, 87,71 e 75,22\% para as temperaturas de 40,55 e $70{ }^{\circ} \mathrm{C}$, respectivamente, com o teor de água variando de 8,425 até 0,170 (b.s.). Observase que a contração volumétrica ocorreu mais intensa nas horas iniciais para todas as temperaturas, sendo que na temperatura de $70{ }^{\circ} \mathrm{C}$ para atingir a mesma taxa de contração volumétrica foi necessário menos tempo em relação às outras temperaturas estudadas; entretanto, a redução do seu volume foi menor dentre as outras temperaturas em que tal fenômeno pode ser explicado em virtude da redução rápida do teor de água em altas temperaturas, o que ocasiona a rápida transição do estado elástico do material para o estado vítreo acarretando uma redução na taxa de encolhimento como foi observado por Paglarini et al., 2012, analisar o fenômeno do encolhimento de abacaxi nas temperaturas de 40,50 e $60{ }^{\circ} \mathrm{C}$.

Os resultados obtidos para difusão líquida para as temperaturas de 40,55 e $70^{\circ} \mathrm{C}$, foram: $1,76 \times 10^{-11}, 4,01 \times 10^{-11} \mathrm{e}$ $8,78 \times 10^{-11} \mathrm{~m}^{2} \mathrm{~s}^{-1}$, respectivamente. Com os resultados obtidos experimentalmente, é possível perceber que os valores do coeficiente de difusão líquida do figo aumentam em função da temperatura e seus resultados corroboram com Silva et al. (2009) que, estudando a secagem em camada fina de banana a 50, 60 e $70^{\circ} \mathrm{C}$ em secador de leito fixo, encontraram valores variando de $3,307 \times 10^{-10}$ a $12,54 \times 10^{-10} \mathrm{~m}^{2} \mathrm{~s}^{-1}$ e Resende et al. (2011) ao estudar a secagem de sementes de pinhão-manso em estufa com ventilação de ar forçado a 30, 40, 50, 60 e 70 ${ }^{\circ} \mathrm{C}$ e obtiveram valores entre $3,93 \times 10^{-10}$ e $9,19 \times 10^{-10} \mathrm{~m}^{2}$ $\mathrm{s}^{-1}$. Segundo Madamba et al. (1996) os valores para difusão líquida de produtos agrícolas estão na ordem de grandeza de $10^{-9}$ a $10^{-11}$.

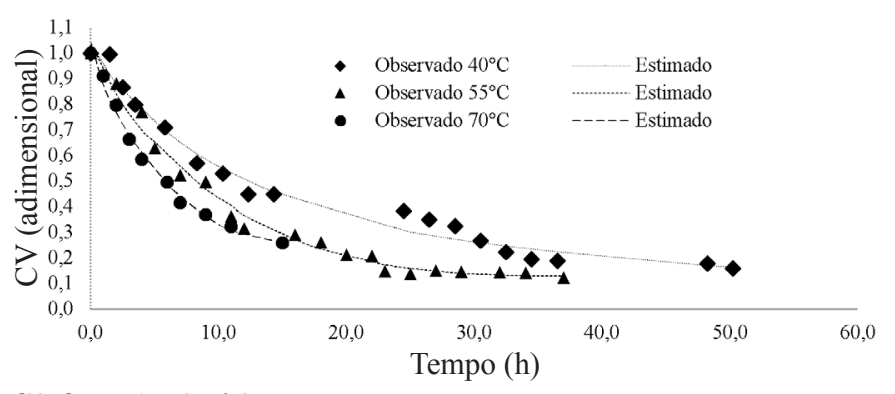

CV - Contração volumétrica

Figura 2. Valores observados e estimados da contração volumétrica para figo pelo modelo polinomial em função do tempo para as temperaturas estudadas 


\section{Conclusões}

1. Os modelos de Midilli e Polinomial foram os que melhor se ajustaram aos dados experimentais das curvas de secagem e contração volumétrica do figo, respectivamente.

2. Pequenos incrementos no valor da temperatura do ar de secagem promovem redução significativa no tempo de secagem dos figos.

3. A redução do teor de água pela secagem influencia na contração volumétrica do figo provocando redução de volume em 84,04; 87,71 e 75,22\% para as temperaturas de 40,55 e $70{ }^{\circ} \mathrm{C}$.

4. A difusão líquida aumenta com a elevação da temperatura apresentando valores entre $1,76 \times 10^{-11} \mathrm{e} 8,78 \times 10^{-11} \mathrm{~m}^{2} \mathrm{~s}^{-1}$.

\section{Literatura Citada}

Alves, G. E.; Isquierdo, E. P.; Borém, F. M.; Siqueira, V. C., Oliveira, P. D.; Andrade, E. T. Cinética de secagem de café natural para diferentes temperaturas e baixa umidade relativa. Coffee Science, v.8, p.238-247, 2013.

Anderson, R. B.; Modification of BET equation. Journal of American Chemical Society, v.68, p.686-691, 1946. http://dx.doi. org/10.1021/ja01208a049

Berg, C. van den. Description of water activity of foods for engineering purposes by means of G.A.B. model sorption. In: Engineering and food. Mckenna, B. M. (ed.) London: Elsevier Applied Science, v.1, 1984. p.311-321.

Boer, J. H. van de. The dynamical character of adsorption. Oxford: Clarendon Press. 1953. 239p.

Borges, S. V.; Mancini, M. C.; Corrêa, J. L. G.; Leite, J. Drying kinetics of bananas by natural convection: Influence of temperature, shape, blanching and cultivar. Ciência e Agrotecnologia, v.35, p.368-376, 2011. http://dx.doi.org/10.1590/S1413-70542011000200019

Brooker, D. B.; Bakker-Arkema, F. W.; Hall. C. W. Drying and storage of grains and oilseeds. New York: Van Nostrand Reinold, 1992. $450 \mathrm{p}$.

Chen, C.; Jayas, D. S. Evaluation of the GAB equation for the isotherms of agricultural products. Transactions of ASAE, v.41, p.1755-1760, 1998. http://dx.doi.org/10.13031/2013.17319

Chen, C.; Morey, V. Equilibrium relativity humidity (ERH) relationships for yellow-dente corn. Transactions of ASAE, v.32, p.999-1006, 1989. http://dx.doi.org/10.13031/2013.31105

Corrêa, P. C.; Machado, P. F.; Andrade, E. T. Cinética de secagem e qualidade de grãos de milho-pipoca. Ciência e Agrotecnologia, v.25, p.134-142, 2001.

Doymaz, I. Sun drying of figs: an experimental study. Journal of Food Engineering, v.71, p.403-407, 2005. http://dx.doi.org/10.1016/j. jfoodeng.2004.11.003

Francisco, V. L. F. S.; Baptistella, C. S. L.; Amaro, A. A.; Fagundes, P. R. S. Evolução e aspectos socioeconômicos da cultura do figo no estado de São Paulo. Informações Econômicas, v.41, p.13-22, 2011.

Goneli, A. L. D.; Corrêa, P. C.; Magalhães, F. E. A.; Baptestini, F.M.; Contração volumétrica e forma dos frutos de mamona durante a secagem. Acta Scientiarum. Agronomy, v.33, p.1-8, 2011. http:// dx.doi.org/10.4025/actasciagron.v33i1.4629
Guggenheim, E. A. Application of statistical mechanics. Gloucestershire: Clarendon Press. 1966. 220p.

IAL - Instituto Adolfo Lutz. Normas analíticas do instituto Adolfo Lutz: Métodos químicos e físicos para análise de alimentos. 3.ed. São Paulo: Adolfo Lutz, v.1. 1985. 332p.

Krokida, M. K.; Maroulis, Z. B. Effect of drying method on shrinkage and porosity. Drying Technology, v.15, p.2441-2458, 1997. http:// dx.doi.org/10.1080/07373939708917369

Leonel, S.; Tecchio, M. A. Épocas de poda e uso da irrigação em figueira 'Roxo de Valinhos' na região de Botucatu, SP. Bragantia, v.69, p.571-580, 2010. http://dx.doi.org/10.1590/S000687052010000300008

Lima, A. G. B. de; Queiroz, M. R.; Nebra, S. A. Simultaneous moisture transport and shrinkage during drying of solids with ellipsoidal configuration. Chemical Engineering Journal, v.86, p.85-93, 2002. http://dx.doi.org/10.1016/S1385-8947(01)00276-5

Madamba, P. S.; Driscoll, R. H.; Buckle, K. A. Thin layer drying characteristics of garlic slices. Journal of Food Engineering, v.29, p.75-97, 1996. http://dx.doi.org/10.1016/0260-8774(95)00062-3

Marinos-Kouriss, D.; Maroulis, Z. B. Transport properties in the drying of solids. In: Mujumdar, A. S. (ed.), Handbook of industrial drying. New York: Marcel Dekker. 1995. v.1, 2.ed., p.113-159.

Mohapatra, D.; Rao, P. S. A thin layer drying model of parboiled wheat. Journal of Food Engineering, v.66, p.513-18, 2005. http:// dx.doi.org/10.1016/j.jfoodeng.2004.04.023

Mohsenin, N. N. Physical properties of plant and animal materials. New York: Gordon and Breach Publishers, 1986. 891p.

Pacco, H. C.; Vigneault, C.; Castro, L. R. de; Menegalli, F. C.; Cortez, L. A. B. Temperature effect on drying kinetics of "Gigante de Valinhos" figs. Journal of Food, Agriculture \& Environment, v.5, p.70-75, 2007.

Paglarini, C. S.; Silva, F. S.; Porto, A. G.; Santos, P.; Piasson, D. Estudo do fenômeno de encolhimento no processo de secagem de fatias de abacaxi pérola. Enciclopédia Biosfera, v.8, p.1914-1926. 2012.

Resende, O.; Ullmann, R.; Siqueira, V. C.; Chaves, T. H.; Ferreira, L. U. Modelagem matemática e difusividade efetiva das sementes de pinhão-manso (Jatropha curcas L.) durante a secagem. Engenharia Agrícola, v.31, p.1123-1135. 2011. http://dx.doi.org/10.1590/ S0100-69162011000600010

Rodrigues, M. G. F.; Corrêa, L. S.; Santos, P. C.; Tulmann Neto, A. Seleção de clones de figueira cv. Roxo de Valinhos formados por gemas irrigadas. Revista Brasileira de Fruticultura, v.34, p. 604611, 2012. http://dx.doi.org/10.1590/S0100-29452012000200036

Silva, A. S.; Melo, K. S.; Alves, N. M. C.; Fernandes, T. K. S.; Farias, P. A. Cinética de secagem em camada fina da banana maçã em secador de leito fixo. Revista Brasileira de Produtos Agroindustriais, v.11, p.129-136, 2009. http://dx.doi.org/10.15871/1517-8595/rbpa. v11n2p129-136

Simão, S. Manual de fruticultura. São Paulo: Ceres, 1971. 530p.

Siqueira, V. C.; Resende, O.; Chaves, T. H. Determination of the volumetric shrinkage in jatropha seeds during drying. Acta Scientiarum Agronomy, v.34, p.231-238. 2012. http://dx.doi. org/10.4025/actasciagron.v34i3.14402

Sousa, S. Obtenção de figos secos por desidratação osmótica e secagem convectiva. Campinas: UNICAMP, 2008. 183p. Tese Doutorado 